\title{
VORSCHAU $02>$
}

\section{ENTWICKLUNG}

Sensoren zur Reifendruckmessung

intelligent steuern

Passive Bauelemente mit Embedded

Systems spezifikationsgerecht entwickeln

Entwicklung von Software für eingebettete Systeme mit kryptologischen Ansätzen

Aktive elektronische Geräuschminderung bei elektrischen Schließsystemen

\section{FORSCHUNG}

Modellierung des dynamischen Verhaltens von Komponenten im Hochvoltbordnetz unter

Berücksichtigung des EMV-Verhaltens

\author{
CAN-, LIN, Flexray-Busse mit einem \\ statt mehreren Testsystemen prüfen \\ Vernetzung von Infotainmentfunktionen \\ in einem digitalen Cockpit \\ Tachomanipulation mit erweiterter \\ Steuergerätekonfiguration verhindern
}

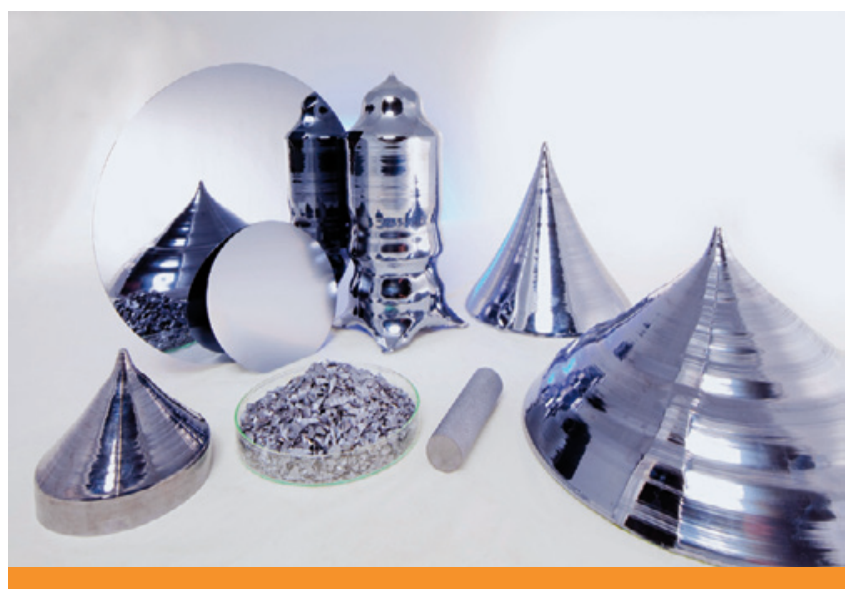

\section{TITELTHEMA}

\section{MATERIALREZEPTE FÜR LEISTUNGSELEKTRONIKEN}

Die Potenziale in der Leistungselektronik liegen in der Erforschung und Entwicklung von Materialkonzepten. Das schließt die fertigungsgerechte Entwicklung mit ein, also auch neue Produktionsverfahren. Mit der galvanischen Abscheidung von Schichten aus Kupfer, Zinn, Nickel und Gold auf Siliziumscheiben steht im Fraunhofer-Institut für Mikroelektronische Schaltungen (IMS) jetzt eine Technik zur Verfügung, mit der integrierte Schaltungen um neue Funktionen erweiterbar sind. Interessant ist, wie sich die Systeme in Richtung besserer Hochstromfähigkeit optimieren lassen.

Was ist mit heutigen Materialrezepten möglich? Wie lassen sich diese produktionsseitig umsetzen? Magna Powertrain beantwortet die Fragen am Beispiel einer elektrischen Achse. Der Zulieferer fokussiert dabei das Elektromotorenkonzept sowie die Invertertechnik. Wie stellt sich Audi diesbezüglich auf? ATZelektronik spricht mit Dr. Christian Allmann, dem Leiter ePerformance bei der Audi Electronic Venture GmbH. In seiner erweiterten Funktion ist er nun auch für die produktionstechnische Umsetzung künftiger Elektrofahrzeugtechnik verantwortlich. 